\title{
Indicadores de Sustentabilidade em Empreendimentos de Mineração
}

\author{
Sâmara Hissa Neiva Aguiar \\ MBA em Perícia, Auditoria e Gestão Ambiental \\ Clarice Neffa Gobbi, \\ Escola Politécnica/UFRJ \\ Manoel Gonçalves Rodrigues \\ Observatório Urbano/UERJ/UN-Habitat \\ Roberto Ricardo Rachid Saab Barbosa Cunha ${ }^{4}$ \\ Observatório Urbano/OUERJ/UN-Habitat
}

\section{RESUMO}

Este artigo propõe apresentar as definições básicas sobre o desenvolvimento sustentável, relatando o desenvolvimento das questões ambientais e sociais do setor de mineração. Traz ainda os principais indicadores de sustentabilidade utilizados pelas maiores empresas de extração mineral do país e qual a metodologia adotada por cada conjunto de indicadores para mensurar o desempenho sustentável das empresas. A pesquisa se caracteriza como descritiva e qualitativa. Utilizou-se a coleta de dados secundários obtidos através das informações disponíveis acerca dos indicadores pesquisados.

Palavras-chave: Qualidade Ambiental; Indicadores; Desenvolvimento Sustentável.

\section{ABSTRACT}

This article proposes to present the basic definitions on sustainable development, reporting the development of environmental and social issues in the mining sector. It also brings the key sustainability indicators used by major mining companies in the country and what the methodology adopted by each set of indicators to measure the sustainability performance of companies. The research is characterized as descriptive and qualitative. We used the collection of secondary data obtained through the available information about the indicators surveyed.

Keywords: Environmental Quality, Indicators, Sustainable Development

\section{INTRODUÇÃO}

Historicamente a extração mineral não foi realizada com os devidos cuidados em relação às questões ambientais, sociais e econômicas. No Brasil, apenas após a partir do último quarto de século, normas e regulamentações foram estabelecidas para impedir a degradação decorrente da extração mineral. A pouca importância 
dada ao meio ambiente e consequentemente para a sociedade pode ser vista ainda hoje nos municípios onde existiam ou ainda existem lavras de metais e pedras preciosas, revelando que a riqueza extraída pouco contribuiu para o desenvolvimento da região.

No Brasil e no mundo, a mineração deixou um legado de passivos, porém este cenário tem se alterado significativamente através da conscientização da sociedade e consequente mudança legal e institucional. A partir dos anos 1990, os marcos de responsabilidade social e ambiental ganharam mais impulso e as atividades de exploração mineral passaram a controlar seus impactos e licenciar seus empreendimentos.

Hoje, mais de 20 anos depois, com a maturidade dos sistemas de gestão ambiental começam a surgir os indicadores de sustentabilidade, caracterizando-se como importantes ferramentas para o desenvolvimento sustentável e reporte de desempenho aos stakeholders (partes interessadas).

Segundo a Comissão Mundial sobre Meio Ambiente através do Relatório Nosso Futuro Comum (1987), o desenvolvimento sustentável é o desenvolvimento capaz de suprir as necessidades da geração atual, sem comprometer a capacidade de atender as necessidades das futuras gerações. À medida que o conceito de desenvolvimento sustentável passou a ser demandado pela sociedade, as empresas buscaram ferramentas que possibilitassem mensurar as ações e inciativas realizadas em prol deste conceito, de forma a reportar seu desempenho aos seus stakeholders.

Em 1997 foi proposto por John Elkington o triple bottom line ou tripé da sustentabilidade, conceito gráfico que enfatiza os aspectos considerados fundamentais para a sustentabilidade: crescimento econômico, equidade social e proteção ao meio ambiente. No tripé estão contidos os aspectos que devem interagir, de forma holística, para satisfazer o conceito.

Anteriormente uma empresa era considerada sustentável se tivesse um bom patrimônio e um lucro sempre crescente. Para um país, o conceito incluía um viés social, já que o desenvolvimento teria que incluir uma repartição da riqueza gerada 
pelo crescimento econômico, seja por meio de mais empregos criados, seja por mais serviços sociais para a população em geral. Esse critério, na maioria das vezes, é medido pelo Produto Interno Bruto (PIB) do país. Porém, para o novo conceito, esta é uma medição limitada. A perna ecológica do tripé trouxe, então, um problema e uma constatação: se os empresários não cuidassem do aspecto ambiental podiam ficar sem matéria-prima e talvez, sem consumidor, além de contribuírem para a destruição do planeta.

De acordo com o tripé da sustentabilidade a empresa é inserida e dependente das esferas econômica, social e ambiental e com estas interage de forma dependente, isto é, se as três esferas estiverem em equilíbrio contribuirão para o equilíbrio e prosperidade da instituição.

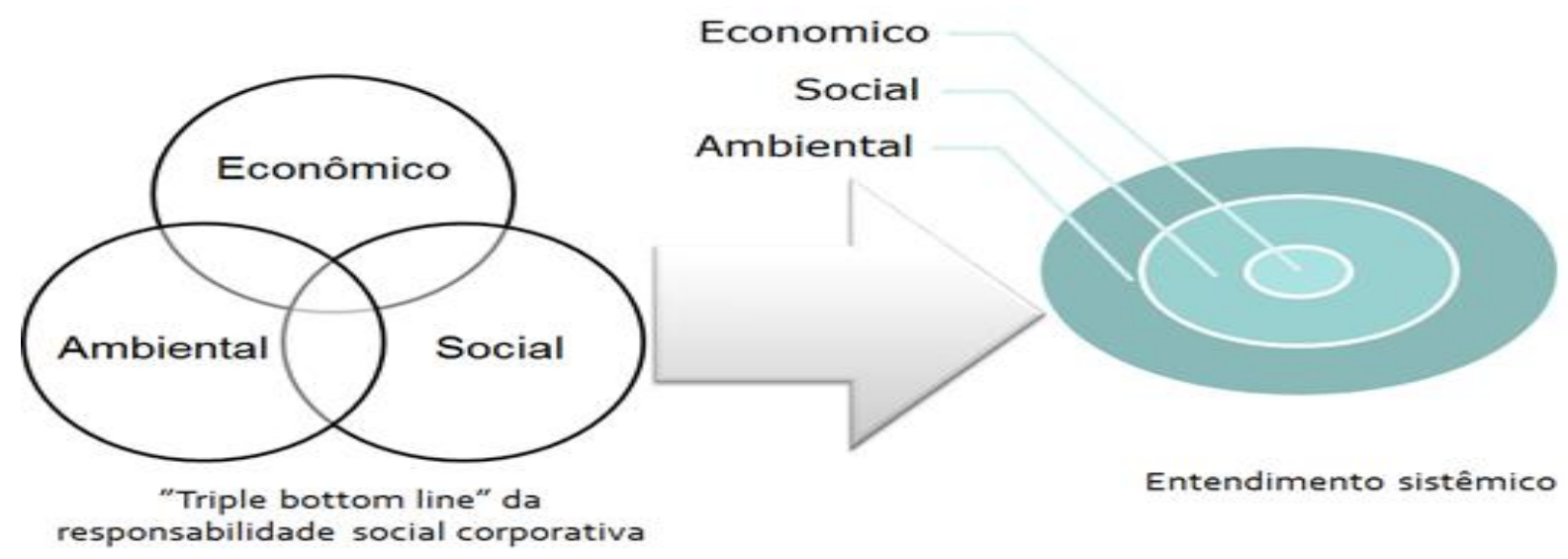

Figura 01 - Conceito do triple bottom line

\section{OBJETIVO GERAL}

O objetivo deste artigo é promover uma reflexão sobre a evolução das questões sociais e ambientais nas empresas de extração mineral e as ferramentas desenvolvidas para mensurar o desempenho destas instituições através dos indicadores de sustentabilidade. 
Uma vez que o conceito de desenvolvimento sustentável era cada vez mais aceito pela sociedade e consequentemente pelas empresas, surgiu a necessidade de avaliar o desempenho das economias com base neste novo conceito e não apenas em indicadores como o PIB. Chegou-se ao consenso de que apenas o PIB não refletia o bem-estar econômico e não permitia avaliar a sustentabilidade do desenvolvimento.

Para aplicar o conceito de desenvolvimento sustentável tornou-se fundamental a definição de indicadores que pudessem dar a medida do desempenho de uma instituição em matéria de sustentabilidade.

Assim, os indicadores surgiram como parâmetros a serem considerados combinados ou isolados. Frequentemente são apresentados nas categorias ambientais, sociais, econômicas e institucionais, normalmente subdivididos em indicadores específicos dessas categorias de forma a atender as nuances das organizações.

Existem diversos conjuntos de indicadores aceitos pelo mercado empresarial, abaixo estão apresentados e descritos os mais comumente utilizados pelas minerações no Brasil:

\section{Global Report Initiative - GRI}

A Global Reporting Initiative (GRI) é uma organização que tem por objetivo compartilhar uma linguagem coerente, para comunicação das organizações com suas partes interessadas. Fundada em 1997, a organização coordena a elaboração multistakeholder de uma estrutura de relatórios de sustentabilidade, que goza de ampla e crescente credibilidade.

A estrutura da GRI contempla orientações quanto ao formato e conteúdo de relatórios de sustentabilidade. No item de conteúdo, um conjunto de protocolos de indicadores é recomendado para as empresas relatarem seu desempenho nas dimensões: econômica, sociedade, práticas trabalhistas e trabalho descente, meio ambiente, respeito aos direitos humanos, e responsabilidade sobre os produtos. Além desses protocolos de indicadores, suplementos setoriais fornecem orientações especificas sobre indicadores mais materiais para diferentes setores da economia.

\section{ISE Bovespa}


O Índice de Sustentabilidade Empresarial (ISE) Bovespa é um índice de bolsa de valores (valoração em moeda corrente de um determinado grupo de ações) composto por ações de empresas com reconhecido comprometimento com a responsabilidade social e a sustentabilidade empresarial, e também atua como promotor das boas práticas no meio empresarial brasileiro.

Essas empresas são selecionadas anualmente através da aplicação de um questionário fundamentado no conceito de "triple bottom line", elaborado e mantido pelo Centro de Estudos em Sustentabilidade da Fundação Getúlio Vargas (GVCes).

Para ser incluída no índice, uma empresa precisa ser uma das 200 ações mais negociadas na bolsa nos doze meses anteriores à reavaliação; ter sido negociada em pelo menos $50 \%$ dos pregões nos doze meses anteriores; e atender aos critérios de sustentabilidade referendados pelo conselho do ISE e avaliados pelo questionário.

O índice é composto por até 40 empresas, sendo definido anualmente com base nos critérios acima, e composto pelas empresas respondentes do questionário. Cabe ressaltar que as informações fornecidas pelas empresas não são verificadas.

Desde sua criação em 2005, até março de 2011, o desempenho da carteira do ISE tem demonstrado grande aderência ao movimento de mercado do benchmark Ibovespa. Após a crise financeira mundial de 2008, a carteira do Ibovespa se distanciou do desempenho do ISE e vem apresentando retornos maiores que o índice de sustentabilidade.

Em outras palavras, a metodologia ISE não demonstrou criação de a (retornos financeiros acima da média), e atraí a atenção apenas de investidores envolvidos diretamente com estratégias de investimento ligadas ao ativismo socioambiental.

- GER - Dimensão Geral

- NAT - Dimensão Natureza do produto

- GOV - Dimensão Governança corporativa

- ECO - Dimensão Econômico-financeira

- $\mathrm{AMB}$ - Dimensão Ambiental Unificado

- SOC - Dimensão Social 


\section{DJSI}

Desenvolvido pela SAM-Group em 1999, o Dow Jones Sustainability Index (DJSI) é um conjunto de índices de bolsa de valores composto por uma carteira selecionada de empresas com demonstrado desempenho extra financeiro nas dimensões social, econômico e ambiental, segundo a definição do índice: "empresas focadas em criar valor aos acionistas no longo prazo, aproveitando oportunidades e gerenciando riscos provenientes de questões sociais, ambientais e econômicas".

O DJSI avalia um universo de empresas públicas com ações negociadas em bolsa de valores e avalia a sustentabilidade corporativa a partir de um sistema de critérios de desempenho socioambiental que inclui indicadores gerais e específicos para cada indústria. A avaliação é feita através de entrevistas com gestores, um questionário enviado às empresas, documentos fornecidos pelas empresas como relatórios anuais e de sustentabilidade e informações da mídia e outros stakeholders. Toda informação declarada pelas empresas é auditada e verificada por uma entidade independente.

Os índice e sub índices têm apresentado retornos financeiros acima da média do mercado, superando a média de mercado ao longo dos anos. Um estudo de 2009 da SAM apresentou que os portfólios de líderes de mercado em sustentabilidade (segundo critérios do índice) tiveram desempenho em média 1,48\% superior a média por ano, enquanto aqueles ativos com pior desempenho em sustentabilidade também tiveram seus ativos valorizados $1,46 \%$ a menos que a média do mercado.

\section{ICMM}

O Conselho Internacional de Metais e Mineração (ICMM - International Council on Mining \& Metals) estabeleceu em 2003 que seu conselho de empresas, colocaria em prática e acompanharia o desempenho em questões sociais, ambientais e de governança, orientado por 10 princípios para o desenvolvimento sustentável. Esses princípios são:

1. Implementar e manter praticas de negócios éticas, e sistemas de governança sólidos;

2. Integrar considerações para o desenvolvimento sustentável no processo de tomada de decisões; 
3. Apoiar os direitos humanos e respeito a culturas, costumes e valores, ao lidar com empregados e outros afetados pelas atividades;

4. Implementar estratégias de gestão de riscos, baseadas em dados válidos e fatos cientificamente comprovados;

5. Buscar a melhoria contínua no desempenho em saúde e segurança;

6. Buscar a melhoria contínua no desempenho ambiental;

7. Contribuir para a conservação da biodiversidade e abordagens integradas no planejamento para o uso da terra;

8. Facilitar e encorajar o projeto, uso, reuso, reciclagem e descarte responsáveis de nossos produtos;

9. Contribuir com o desenvolvimento social, econômico e institucional das comunidades em que operamos;

10. Implementar estratégias efetivas e transparentes com os stakeholders para engajamento, comunicação e relatórios auditados.

Mais recentemente (concluído em 2010), o ICMM em parceria com o Global Reporting Initiative (GRI) confeccionou o suplemento setorial para a mineração (MMSS) do G3 - o guia de orientações para elaboração de relatórios de sustentabilidade organizado pelo GRI a partir de consultas a painéis de stakeholders. Esse suplemento inclui observações específicas e um conjunto de indicadores próprio do setor de mineração, estruturado conforme o padrão do GRI.

\section{Pacto Global}

Uma iniciativa da Organização das Nações Unidas (ONU), o Pacto Global é uma iniciativa de alinhamento das práticas empresariais com dez princípios universalmente aceitos nas áreas de direitos humanos, trabalho, meio-ambiente e combate a corrupção. Esses princípios são:

Direitos Humanos

1. As empresas devem apoiar e respeitar a proteção de direitos humanos reconhecidos internacionalmente;

2. Assegurar-se de sua não participação em violações destes direitos.

$\underline{\text { Trabalho }}$ 
3. As empresas devem apoiar a liberdade de associação e o reconhecimento efetivo do direito à negociação coletiva;

4. A eliminação de todas as formas de trabalho forçado ou compulsório;

5. A abolição efetiva do trabalho infantil;

6. Eliminar a discriminação no emprego.

Meio ambiente

7. As empresas devem apoiar uma abordagem preventiva aos desafios ambientais;

8. Desenvolver iniciativas para promover maior responsabilidade ambiental;

9. Incentivar o desenvolvimento e difusão de tecnologias ambientalmente amigáveis.

\section{Combate à corrupção}

10. As empresas devem combater a corrupção em todas as suas formas, inclusive extorsão e propina.

Os objetivos do Pacto global são divulgar e popularizar os dez princípios entre as atividades corporativas; e catalisar ações em apoio a objetivos mais abrangentes como os Objetivos de Desenvolvimento do Milênio das Nações Unidas.

\section{Indicadores Ethos}

Desenvolvidos e revisados pelo Instituto Ethos desde 2000, os indicadores Ethos são um conjunto de questões para auto avaliação das empresas em questões de responsabilidade socioambiental corporativa, e têm por objetivo orientar as empresas a implementarem práticas sustentáveis.

O questionário de avaliação da empresa está dividido em sete grandes temas:

1. Valores, Transparência e Governança;

2. Público Interno;

3. Meio Ambiente;

4. Fornecedores;

5. Consumidores e clientes;

6. Comunidade;

7. Governo e sociedade. 
Esses temas são enfocados por três tipos de indicadores:

Indicador de Profundidade: Permite avaliar o estágio atual da gestão da empresa. É representado por quatro quadros contíguos apresentando estágios de determinada prática, cujo nível de desempenho evolui da esquerda para a direita, o que permite à empresa situar-se facilmente na escala. $O$ quadro mais à direita corresponde ao melhor desempenho naquela prática e pressupõe que a empresa já atingiu um nível de excelência naquele indicador.

Indicador Binário: É composto por questões binárias (sim ou não) relacionadas ao primeiro grupo de perguntas. Contém elementos de validação e aprofundamento da avaliação sobre o estágio em que a empresa se identifica e contribui para a compreensão de quais práticas devem ser incorporadas à gestão dos negócios.

Indicador Quantitativo: Propõe o levantamento sistemático de dados (que podem ser avaliados segundo séries anuais e cruzados com outros). Nem todos os indicadores apresentam dados quantitativos. No entanto, os que existem, devem ser precisamente levantados, pois serão úteis, principalmente no monitoramento interno da empresa.

O Instituto Ethos, em parceria com o Instituto Brasileiro de Mineração - IBRAM, desenvolveu o questionário setorial de Mineração que propõe uma auto avaliação mais profunda e focada nas questões relevantes para o setor. Este questionário compreende 40 questões aplicadas especificamente a empresas do setor de extração mineral.

IFC

A International Finance Corporation - IFC é um braço do Banco Mundial que fornece consultoria e investimentos para o setor privado em países em desenvolvimento.

Os padrões de desempenho IFC são documentos que ajudam a organização e seus clientes a administrar e melhorar o desempenho social e ambiental, adotando uma abordagem baseada em resultados. Os padrões de desempenho também oferecem uma base sólida sobre a qual os clientes podem melhorar a sustentabilidade das operações. Esses padrões são:

1. Sistemas de avaliação e gestão socioambiental;

2. Condições de emprego e trabalho; 
3. Prevenção e redução da poluição;

4. Saúde e segurança da comunidade;

5. Aquisição da terra e ressarcimento involuntário;

6. Preservação da biodiversidade e manejo sustentável dos recursos naturais;

7. Povos indígenas;

8. Patrimônio cultural.

Cada padrão conta com um descritivo detalhado, indicadores e métodos de abordagem para cada projeto e fator analisado.

Os padrões de desempenho do IFC constituem a base de avaliação da sustentabilidade dos Princípios do Equador, um conjunto de princípios adotados por bancos de crédito para avaliar e gerenciar riscos sociais e ambientais nos projetos investidos.

\section{GS Sustain}

GS Sustain é uma estratégia de investimentos da Goldman Sachs, que considera questões de desempenho socioambiental e de governança (Environmental, Social and Governance - ESG) juntamente com uma análise de atratividade da indústria e desempenho financeiro.

A partir de uma avaliação nessas três dimensões (gestão de ESG, atratividade da indústria e retorno sobre capital), empresas em cada setor com melhor desempenho são selecionadas e nomeadas "líderes GS Sustain".

A metodologia de avaliação de ESG conta com vinte e quatro itens (sendo 4 exclusivos para o setor de mineração) distribuído nas dimensões de Governança, Meio Ambiente e Social, sendo esta última subdividida nas categorias stakeholders, Colaboradores e Liderança. Cada critério é avaliado de uma escala de 1 a 5 . Do universo de empresas avaliadas pela Goldman Sachs, as $25 \%$ melhor avaliadas são listadas para avaliação posterior quanto à atratividade do mercado e desempenho financeiro.

\section{CONSIDERAÇOES FINAIS}


A partir dos conjuntos de indicadores pesquisados, pôde-se verificar que as empresas em especial as do setor de mineração que adotam um ou mais conjuntos de indicadores possuem em geral um desempenho melhor de mercado.

Cabe ressaltar que as questões de meio ambiente, trabalho e renda, comunicação com as partes interessadas e governança estão presentes em diversos conjuntos de indicadores e ao reportar ou atender um conjunto especifico a instituição estará atendendo a outros indicadores de outros conjuntos.

Conforme ilustrado na figura abaixo o ganho resultante da busca pela sustentabilidade de uma instituição será decorrente do amadurecimento de diversos aspectos cruciais para um bom resultado financeiro, econômico e social.

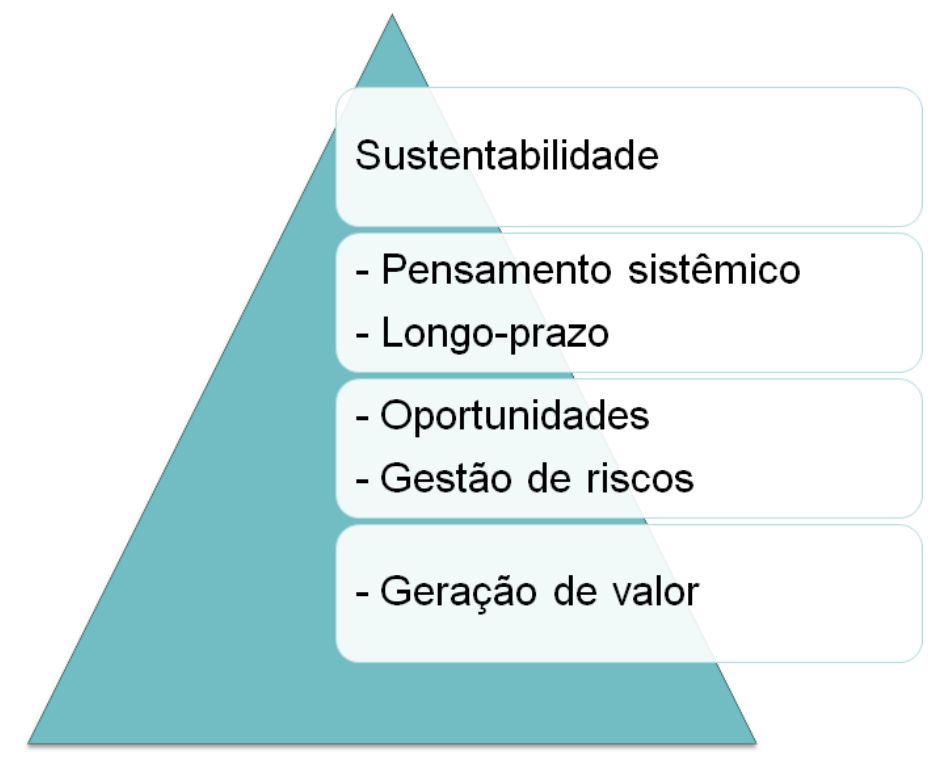

Figura 3 - Aspectos da Sustentabilidade

As empresas ao demonstrarem transparência e controle em suas operações e projetos em relações as questões ambientais, sociais e econômicas, garantem aos investidores maior segurança no investimento, principalmente se considerarmos que as empresas de extração mineral em geral são frequentemente alvo de criticas e especulações pela sociedade.

Outros benefícios como foco no longo-prazo, maior acesso a financiamentos, forte governança corporativa, valorização da marca e reputação e ganhos em eficiência operacional também podem ser inseridos como benefícios as instituições que trabalham com a premissa do desenvolvimento sustentável. 


\section{REFERÊNCIAS}

SAM SUSTAINABILITY INVESTING E DOW JONES INDEXES: Brochura Dow Jones Sustainability Indexes. Disponível em: <http://www.djindexes.com/mdsidx/downloads/brochure_info/Dow_Jones_Sustainabil ity_Indexes_Brochure.pdf > Acesso dia 22/03/2011

SAM SUSTAINABILITY INVESTING: Relação de empresas convidadas para o DJSI. Disponível em: <http://www.sustainabilityindex.com/djsi pdf/SAM DJSI InvitedCompanies 2010.xls > Acesso dia 22/03/2011

ISE BOVESPA, Evolução do desempenho do ISE Bovespa. Disponível em: $<$ http://www.bmfbovespa.com.br/indices/ResumoEvolucaoDiaria.aspx?Indice=ISE\&id ioma $=$ pt-br $>$ Acesso dia 22/03/2011

GOLDMAN SACHS. Estudo GS Sustain para Mineração. Documento disponibilizado com solicitação

GLOBAL REPORTING INITIATIVE (Inglês). Disponível em: <http://www.globalreporting.org $>$. Acesso dia: 23/03/2011

GLOBAL REPORTING INITIATIVE (Português). Disponível em: $<$ http://www.globalreporting.org/Home/LanguageBar/PortugueseLanguagePage.htm >. Acesso dia: 23/03/2011

GLOBAL REPORTING INITIATIVE. Suplemento Setorial de Mineração e Metais. Disponível

em:

$<$ http://www.globalreporting.org/ReportingFramework/SectorSupplements/MiningAnd Metals/MiningAndMetals.htm>. Acesso dia: 23/03/2011

BM\&F BOVESPA. Página oficial do índice ISE Bovespa. Disponível em: $<$ http://www.bmfbovespa.com.br/indices/Resumolndice.aspx?Indice=ISE\&Opcao=0\& idioma=pt-br $>$. Acesso dia: 23/03/2011

BM\&F BOVESPA. Apresentação Institucional do ISE Bovespa (Inclui informações sobre desempenho acumulado da carteira de novembro/2005 até janeiro/2010). Disponível

em: $<$ http://www.bmfbovespa.com.br/Indices/download/ResumolSENovo.pdf $>$. Acesso dia: 23/03/2011 
BM\&F BOVESPA. Metodologia de cálculo e composição da carteira. Disponível em: <http://www.bovespa.com.br/Pdf/Indices/ISE.pdf>. Acesso dia: 23/03/2011

BM\&F BOVESPA. Questionários de avaliação. Disponível em: $<$ http://www.bmfbovespa.com.br/indices/ResumoQuestionariolSE.aspx?ldioma=pt$\underline{b r}>$. Acesso dia: 23/03/2011

DOW JONES SUSTAINABILITY (em inglês). Disponível em: <http://www.sustainability-index.com/>. Acesso dia: 23/03/2011

DOW JONES SUSTAINABILITY. General Questionnaire (em inglês). Disponível em: $<$ http://www.sustainabilityindexes.com/djsi pdf/general questionnaire 2009.pdf>. Acesso dia 23/03/2011

SAM GROUP. Estudo sobre o a da sustentabilidade (em inglês). Disponível em: $<$ http://www.sam-

group.com/downloads/about/sam press releases/090713 MR Alpha from Sustain ability e.pdf>. Acesso dia: 23/03/2011

DOW JONES SUSTEINABILITY. Livros de guia sobre as metodologias dos índices (em inglês). Disponível em: < http://www.sustainabilityindex.com/07 htmle/publications/guidebooks.html>. Acesso dia: 23/03/2011

INTERNATIONAL COUNCIL ON MINING \& METALS - ICMM. Página dos dez princípios do ICMM (em inglês). Disponível em: <http://www.icmm.com/ourwork/sustainable-development-framework/10-principles>. Acesso dia: 23/03/2011 INTERNATIONAL COUNCIL ON MINING \& METALS- ICMM . Declarações de posicionamento e esclarecimentos sobre alguns princípios (em inglês). Disponível em: <http://www.icmm.com/our-work/sustainable-developmentframework/position-statements $>$. Acesso dia: 23/03/2011

GLOBAL COMPACT. Página internacional do Pacto Global (Global Compact Inglês). Disponível em: <http://www.unglobalcompact.org>. Acesso dia: 23/03/2011

PACTO GLOBAL. Rede brasileira do Pacto Global. Disponível em: <http://www.pactoglobal.org.br>. Acesso dia: 09/07/2010

PACTO GLOBAL. Dez princípios do Pacto Global. Disponível em: <http://www.pactoglobal.org.br/dezPrincipios.aspx>. Acesso dia: 09/07/2010 
INSTITUTO ETHOS. Página de downloads de questionários e referências aos Indicadores

Ethos.

Disponível

em:

$<$ http://www.uniethos.org.br/docs/conceitos praticas/indicadores/download/>.

Acesso dia: 23/03/2011

INSTITUTO ETHOS. Questionário Ethos. Disponível em:<http://indicadores.ethos.org.br>. Acesso dia: 23/03/2011

INSTITUTO ETHOS. Autodiagnóstico setorial de mineração. Disponível em: $<$ http://www.uniethos.org.br/docs/conceitos praticas/indicadores/questionario/minera cao.pdf>. Acesso dia: 23/03/2011

INTERNACIONAL FINANCE CORPORATION - IFC. Padrões de desempenho IFC. Disponível

em:

$<$ http://www.ifc.org/ifcext/sustainability.nsf/Content/PerformanceStandards>. Acesso dia: 23/03/2011

INTERNACIONAL FINANCE CORPORATION - IFC. Orientações do IFC para meio ambiente, saúde e segurança (EHS - Inglês). Disponível em: $<$ http://www.ifc.org/ifcext/sustainability.nsf/Content/EHSGuidelines>. Acesso dia: 23/03/2011

INTERNACIONAL FINANCE CORPORATION - IFC. Orientações de EHS do IFC para mineração (Inglês). Disponível em:

$<$ http://www.ifc.org/ifcext/sustainability.nsf/AttachmentsByTitle/gui EHSGuidelines20 07 Mining/\$FILE/Final+-+Mining.pdf>. Acesso dia: 23/03/2011

GS SUSTAIN. Disponível em: <http://www2.goldmansachs.com/ideas/environmentand-energy/goldman-sachs/gs-sustain/index.html>. Acesso dia: 23/03/2011

INSTITUTO ETHOS. Autodiagnóstico setorial de mineração. Disponível em: $<$ http://www.uniethos.org.br/docs/conceitos_praticas/indicadores/questionario/minera cao.pdf>. Acesso dia: 23/03/2011

WIKIPEDIA. Desenvolvimento Sustentavel. Disponivel em: $<$ http://pt.wikipedia.org/wiki/Desenvolvimento sustent\%C3\%A1vel>. Acesso dia: 23/03/2011

CONSELHO EMPRESARIAL BRASILEIRO PARA O DESENVOLVIMENTO SUSTENTÁVEL. Disponivel em: < http://www.cebds.org.br>. Acesso dia: 01/08/2011 
MINISTÉRIO DO DESENVOLVIMENTO INDÚSTRIA E COMÉRCIO EXTERIOR. Disponivel em:< http://www.mdic.gov.br/arquivos/dwnl 1201279825.pdf>. Acesso dia: 01/09/2010

FINANCIAL MANAGEMENT SERVICE. Treasury Reporting Rates of Exchange as of June 30, 2011. Disponível em: http://www.fms.treas.gov/intn.html. Acesso em $\underline{19 / 07 / 2011}$

AGÊNCIA AMERICANA DE PROTEÇÃO AMBIENTAL. Disponível em: $<$ http://www.epa.gov/>. Acesso em: 21/07/2011

REDE DE SUSTENTABILIDADE. O que é sustentabilidade? Disponivel em: <http://www.sustentabilidade.org.br/>. Acesso em: 21/07/2011

WIKIPEDIA. $\quad$ Sustentabilidade. Disponivel em:< http://pt.wikipedia.org/wiki/Sustentabilidade>. Acesso em: 21/07/2011

SEMANA DO MEIO AMBIENTE. Triple bottom line" ou tripé da sustentabilidade. Disponivel em: <http://semanademeioambiente.blogspot.com/2008/03/triple-bottomline-ou-trip-da.html>. Acesso em: 21/07/2011

ÉPOCA NEGOCIOS. A Triple Botton Line é uma boa idéia? Disponivel em: < http://colunas.epocanegocios.globo.com/ideiaseinovacao/2009/07/15/a-triple-bottomline-e-uma-boa-ideia/>. Acesso em: 21/07/2011 